\title{
Social Isolation and Loneliness
}

J de Jong Gierveld, Netherlands Interdisciplinary Demographic Institute, The Hague, The Netherlands

TG van Tilburg, VU University Amsterdam, Amsterdam, The Netherlands

(C) 2016 Elsevier Inc. All rights reserved.

\section{Glossary}

Loneliness A situation subjectively experienced by an individual as one where there is an unpleasant or inadmissible lack of (quality of) certain relationships. This includes situations in which the number of existing relationships is smaller than is considered desirable, as well as situations where the intimacy one wishes for has not been realized.

Loneliness; emotional type The part of loneliness that stems from the absence of an intimate figure or a close emotional attachment.
Loneliness; social type The part of loneliness that stems from the absence of a broader group of contacts. Social isolation The objective characteristics of a situation of a reduced social network of personal relationships. Social network The objective characteristics (size, composition, and functioning) of the group of kin and nonkin people who are important for the person and with whom one is in more or less frequent contact.

\section{Social Isolation and Loneliness: Different Concepts}

Social isolation concerns the objective and quantifiable characteristics of a situation and refers to a reduced network of kin and nonkin relationships. The central question here is: To what extent is he or she alone? There is a continuum running from social isolation at the one hand to social participation at the other. Persons with a very small number of ties with others are, by definition, socially isolated. Younger and older persons may be socially isolated. Examples of socially isolated persons encompass the student Tom in his first year at the university, living on a campus far away from his family and his former school friends; and the 70-year-old painter Paul, who after many years of living and working in New York, moved to a cottage near the sea coast, cut off by his living in a remote environment. Common characteristics of socially isolated individuals involve the near absence of relationships with others. These situations are defined as situations of social isolation.

Are these people lonely? It is not known. Loneliness is but one of the possible outcomes of the subjective evaluation of a situation characterized by a small number of relationships. Socially isolated persons are not necessarily lonely, and lonely persons are not necessarily socially isolated in the objective sense. Loneliness is subjective, and is a negative experience of an imbalance between realized relationships and the level of desired relationships. Even in sharing personal experiences it is sometimes embarrassing for lonely people to confess their loneliness. Most people in Western societies are oriented toward successful careers, for example, in jobs and social relationships, and it is taboo to talk about negative feelings including loneliness.

\section{Social Isolation: Kernel Characteristics}

Social isolation and loneliness are different concepts, but research has shown that socially isolated persons run a great risk of becoming lonely. Generally speaking, socially isolated persons are often very lonely. Adults who have large social networks are rarely lonely. Therefore, being surrounded by a social network of meaningful personal relationships is of crucial importance for feeling socially embedded and for alleviation of loneliness. Having at least three or four intimate social ties provides the greatest protection against loneliness; the protection provided by additional relationships has only a small additional impact. The size of the social network is important, but many other aspects of the social network are worth mentioning in this context too, such as the composition and functioning of the network. A social network composed of varying types of relationships (e.g., both friends and family) is most helpful in alleviating loneliness. This is especially so when the network is supportive; that is, network members exchange emotional support and practical help.

\section{Loneliness: Kernel Characteristics}

Discussions about loneliness date back to ancient times, when philosophers wrote primarily about 'positive loneliness.' This type of loneliness is seen as a voluntary withdrawal from the daily hassles of life and oriented toward higher goals, such as reflection, meditation, and communication with God. In more recent times, the philosopher Moustakas (1961) differentiates between positive and negative types of loneliness. He describes the positive type of 'existential loneliness' as an inevitable part of human experience, involving periods of self-confrontation, but at the end providing an avenue for self-growth. This positive loneliness is in contrast to the negative type of 'loneliness anxiety,' resulting from a basic alienation from other people.

Thanks to the efforts of Peplau and Perlman (1982), the conceptualization of loneliness as a psychological construct became more or less standardized. At the end of the 1970s, they brought together researchers who studied loneliness from several regions of the world and edited an authoritative book. Stemming from the publication of that volume, empirical 
research into social isolation and loneliness has relied on similar terminology, definitions, measurement instruments, and theoretical ideas. Included in this common body of knowledge is the concept of loneliness as the manner in which individuals subjectively experience and evaluate a lack of communication with other people. This concept of loneliness is adopted widely in research.

The discrepancy subjectively experienced between the desire for personal relationships and actually realized relationships - feelings of missing certain personal relationships - is of crucial importance for the onset and continuation of loneliness. Most researchers use this cognitive discrepancy approach to investigate loneliness. In this context, Russell et al. (2012) showed that students who reported having fewer close friendships than they desired were most likely to be lonely; the student Tom referred to earlier might be one of them. Students who reported a fit between the desired and actual numbers of close friends were least lonely. This brings us to the definition of loneliness:

Loneliness is a situation experienced by the individual as one where there is an unpleasant or inadmissible lack of (quality of) certain relationships. This includes situations in which the number of existing relationships is smaller than is considered desirable, as well as situations where the intimacy one wishes for has not been realized. (De Jong Gierveld, 1987, p. 120)

Feeling lonely is accompanied by feeling emptiness and rejection. The opposite of loneliness is belongingness or feeling embedded. So, a crucial question here is: Does a person wish to have contact with few or with many people? Does a person wish to be intimate with close social partners? Or maybe, does this person wish to be alone, to have only few social contacts? An example of the latter is a person who opts for privacy as a means toward avoiding undesired social contacts. The painter Paul in our example might be such a person, wishing to be alone, due to his passion for painting.

Most people wish to have at least one social contact in whom they can confide their personal worries and feelings. A romantic partner, an adult child, or a best friend is most frequently identified as such an intimate figure. If such a confidant is missing, the risk of loneliness increases. This type of loneliness is designated by Weiss (1973) as emotional loneliness. Feelings of missing related to a broader group of contacts or an engaging social network (e.g., peripheral kin, casual friends, colleagues, and neighbors) is named as the social type of loneliness. Some people are especially prone to emotional loneliness, others to social loneliness, but it is the combination of emotional and social loneliness that leads to the most intense feelings of loneliness.

\section{Individual-Level Risk Factors for Social Isolation and Loneliness}

In discussing risk factors for the onset and continuation of loneliness the theoretical model of Hawkley et al. (2008) is followed, differentiating between distal (age and gender) and structural factors (income and education) that operate through proximal factors (health) to affect social isolation (encompassing the size, composition, and functioning of the social network) and consequently the subjective evaluation of the size and functioning of the network, i.e., social and emotional loneliness (Figure 1).

\section{Gender}

Despite the fact that women typically have larger social networks than men, women report significantly higher levels of loneliness than men. This gender difference is associated with women's higher risks of becoming widowed. In multivariate analyses controlling for marital status, partner history, and other factors such as health, gender differences in loneliness are often not observed.

\section{Age}

Becoming old is often equated with becoming socially isolated and lonely. Results of survey research, summarized by Dykstra (2009), suggest that $20-30 \%$ of the middle-aged and

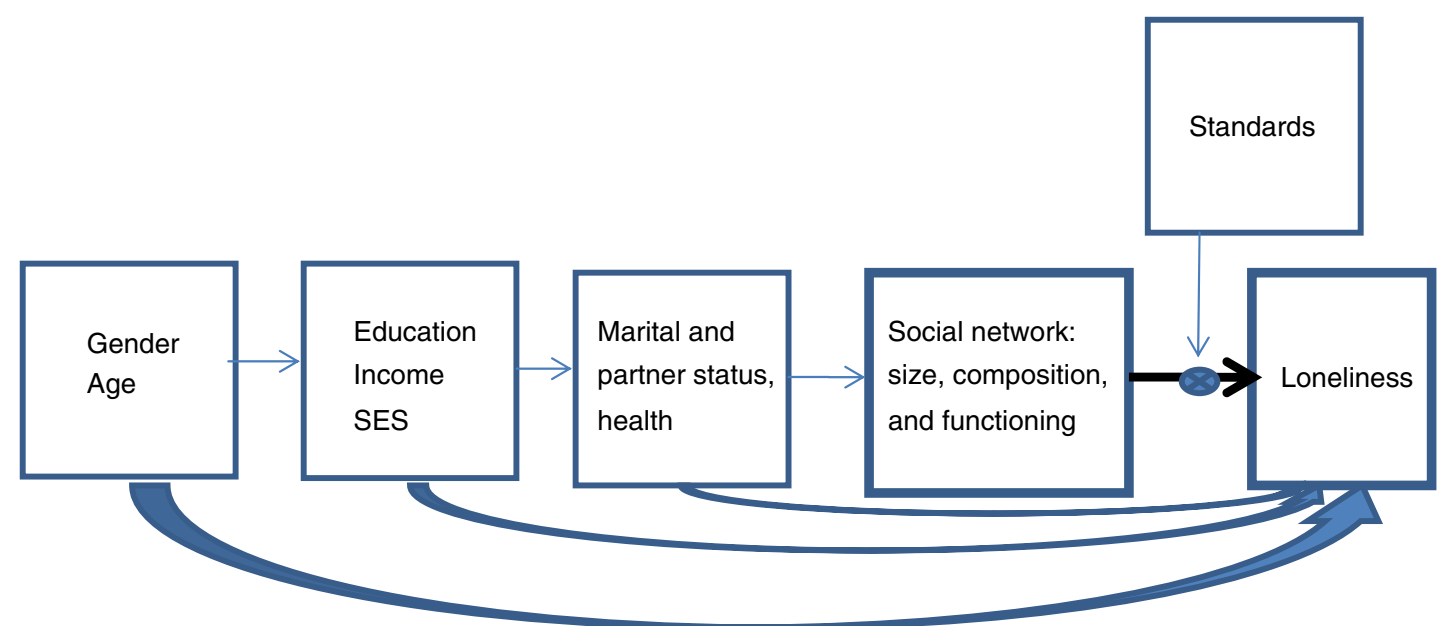

Figure 1 Individual level risk factors affecting social network size, composition and functioning $\rightarrow$ social isolation vs. social participation) and Ioneliness $(\rightarrow$ loneliness vs. social embedment). 
young-old adults report moderate or serious loneliness. Loneliness is more common among adults aged 80 years or older: $40-50 \%$ of adults more than 80 years of age report they often feel lonely. Age is strongly associated with (deteriorating) health: older adults are more frequently confronted with long-term health problems and disabilities that diminish the possibility for maintaining optimal contact with members of their social network and increase the risk of loneliness. Indeed, health and marital status account for many observed age difference in loneliness. When studies also consider health, marital status, and other factors, age differences in loneliness are small.

\section{Educational Level, Income, and Socioeconomic Status}

A lower educational level, economic hardship, and poverty might increase social isolation and loneliness. Research by Scharf et al. (2005) in deprived urban neighborhoods in the United Kingdom found large proportions of the population prone to social isolation and loneliness. The risk of being affected by multiple forms of social isolation and loneliness was greatest for those belonging to minority ethnic groups and aged 75 years and over.

\section{Health}

Poor health and having functional limitations are related to reduced social participation in the community, diminished social contacts with friends and relatives, and poor relationship quality. Those who are in poor health, whether this is measured objectively or subjectively, tend to report higher levels of loneliness.

\section{Marital and Partner Status}

One of the most important factors affecting the prevalence of loneliness is the marital and romantic partner status of adults. Loneliness is significantly more intense among adults who (recently) lost their partners through divorce or death than among married persons. The majority of widowed adults live in a one-person household. Individuals who live alone must seek their social support from people outside the household. Eliciting support from outside the household requires effort and perseverance, and even then, a successful exchange of emotional and instrumental support is not guaranteed. Research has repeatedly shown the protective effect of an intimate spouse or a romantic partner bond on the physical, financial, and mental well-being of both men and women.

The quality of the partner bond is of importance too. Married adults who are disappointed in the quality of their relationship experience more intense feelings of loneliness as compared to married adults who are satisfied with the quality of their partner bond. De Jong Gierveld et al. (2009) found that reciprocal exchange of emotional support, having frequent conversations, the absence of disagreement, and experiencing the sexual relationship as pleasant are of utmost importance to guarantee the alleviation of loneliness within marriage. Remarriage or starting unmarried cohabitation after widowhood or divorce, tends to protect against loneliness and helps to resolve social isolation to a certain extent.

\section{Relationships with Children and Other Kin}

Older adults who have close relationships with children, siblings, and other kin report significantly lower levels of loneliness than older adults with only a few or no kin relationships.

The centrality of the parent-child bond in people's lives is undisputed. Adult children can be an important source of companionship, closeness, and sharing for older adults, especially among older adults who are alone. Routasalo et al. (2006) showed that loneliness is associated with the frequency of contact with children; loneliness is especially associated with expectations regarding the frequency of contact and support exchanges with children and consequently with older adults' satisfaction with their grown children.

In countries where filial norms are dominant characteristics of the familial bonds between generations, older adults may rely on coresidence with an adult child; the child may feel obligated to take care of the needs of the older generation. Changing societal values and migration are among the factors that decrease the incidence of coresidence, decrease social participation, and weaken the patterns of support exchanges within the family. Research by Chen and Short (2008) among Chinese older adults showed that filial support is decreasing, contributing to Chinese older adults' social isolation and loneliness.

Childless adults and adults who are dissatisfied with the contact they have with their children must rely on other family members or nonkin network members for companionship and social support. Older adults who do not develop such ties may experience social isolation and loneliness.

\section{Relationships with Friends and Acquaintances}

Relationships with friends, colleagues, and other nonkin serve to connect people to circles outside the family. The benefits of belonging to a social network that includes nonkin can lower the risks of social loneliness (Cacioppo and Patrick, 2009); Russell et al. (2012) demonstrated that best friends can step in and function as confidants and help alleviate emotional loneliness, in particular for never partnered or childless adults. Involvement in formal organizations is another source of social connections. Church attendance, activities in formal organizations, and volunteer work bring people together and are a means to help prevent or combat loneliness.

\section{The Prevalence of Loneliness and Country-Level Risk Factors for Social Isolation and Loneliness}

Approximately $8 \%$ of the adult population in Western countries experience intense loneliness, and another $20 \%$ experience moderate or mild loneliness. Loneliness is present in all segments of the population; it is a phenomenon known by (previous) personal experience by most adults. Loneliness is in many cases a temporary phenomenon, but sometimes persons are confronted with enduring loneliness. The prevalence of 
loneliness differs according to socio-demographic and many other characteristics of the population.

Levels of loneliness in the United States, Canada, Australia, and Western and Northern Europe are in the same range, although risk factors differ (Van Tilburg et al., 2004). However, research has shown that other countries and regions of the world differ significantly in levels of loneliness. Jylhä and Jokela (1990) found that inhabitants of countries that score high on filial values are not necessarily protected against loneliness. Other factors, such as the prevalence and acceptance of living in small households - either living alone or as a couple - are important as well. In this context, research has shown that older adults in Mediterranean Europe, characterized by having high hopes for frequent relationships with children, siblings and others, have higher levels of loneliness on average than older adults with modest hopes in Northern Europe.

Socially deprived regions, and countries with sharp discrepancies between impoverished inhabitants and more affluent persons have higher incidences of loneliness than countries with a more equitable welfare and health system. In investigating the causes and determinants of loneliness the differences in cultural values and norms, and in the socioeconomic and welfare systems that affect variations in loneliness have to be taken into account.

\section{Social Isolation and Loneliness as Risk Factors for Poor Health and Mortality}

A deficient social network as well as loneliness have serious consequences for future health outcomes. Via both retrospective and prospective surveys it is shown that loneliness predicts poorer physical health, depressive symptoms, alcoholism, and suicidal thinking. A meta-analysis by Holt-Lunstad et al. (2010) showed that a small social network, a shortage of support received from network members, and especially experiencing intense feelings of loneliness are decisive for early mortality among older adults.

\section{Loneliness-Reduction Strategies}

Research has identified three main ways to cope with loneliness: reducing the perceived discrepancy between actual and desired relationships by (1) increasing the number and quality of the relationships to the desired level, (2) decreasing the standards held for the size and functioning of relationships to the level of reality, and (3) reducing the consequences of the discrepancy, for example, by accepting these feelings or by viewing subjectively perceived loneliness in perspective.

In cases of severe loneliness and a small or not optimally functioning social network or in cases of severe disability or chronic illness, social contacts are needed to provide support and guidance to overcome loneliness. Volunteer organizations may step in by arranging places where people can meet other people, and by organizing regular visits to sick and disabled adults in their homes and enhancing their support system. Additionally, professional interventions have been oriented toward improving social skills, and reaching and motivating people to participate in community therapeutic settings.

The effectiveness of loneliness interventions is not well established and must be improved. Evaluation studies indicate that interventions are effective sometimes and only under particular circumstances. For example, arranging meeting places may work for socially lonely individuals who have social skills, but not for emotionally lonely people who grieve the loss of a partner. Effective interventions require intensive action and often reach small numbers of people, whereas the high loneliness prevalence asks for large-scale approaches; internet usage and internet-based video communications might help to overcome this problem.

See also: Bereavement and Grief. Divorce and Parental Separation. Love and Intimacy. Marriage, Romantic Relationships, and Health. Poverty and Mental Health. Siblings. Singles and Mental Health. Social Support and Mental Health. The Stigma of Mental IIIness

\section{References}

Cacioppo, J.T., Patrick, W., 2009. Loneliness: Human Nature and the Need for Social Connection. New York, NY: W. W. Norton \& Company.

Chen, F., Short, S.E., 2008. Household context and subjective well-being among the oldest old in China. Journal of Family Issues 29 (10), 1379-1403.

De Jong Gierveld, J., 1987. Developing and testing a model of loneliness. Journal of Personality and Social Psychology 53, 119-128.

De Jong Gierveld, J., Broese van Groenou, M., Hoogendoorn, A.W., Smit, J.H., 2009. Quality of marriages in later life and emotional and social loneliness. Journals of Gerontology 64B, 497-506.

Dykstra, P.A., 2009. Older adult Ioneliness: Myths and realities. European Journal of Ageing 6 (2), 91-100.

Hawkley, L.C., Hughes, M.E., Waite, L.J., et al., 2008. From social structural factors to perceptions of relationship quality and Ioneliness: The Chicago health, aging, and social relations Study. Journal of Gerontology 63B (6), S375-S384.

Holt-Lunstad, J., Smith, T.B., Layton, J.B., 2010. Social relationships and mortality risk: A meta-analytic review. PLoS Medicine 7 (7), 1-19.

Jylhä, M., Jokela, J., 1990. Individual experiences as cultural: A cross-cultural study on Ioneliness among the elderly. Ageing and Society 10 (2), 295-315.

Moustakas, C.E., 1961. Loneliness. New York, NY: Prentice Hall.

Peplau, L.A., Perlman, D. (Eds.), 1982. Loneliness, a Sourcebook of Current Theory, Research and Therapy. New York, NY: Wiley.

Routasalo, P.E., Savikko, N., Tilvis, R.S., Strandberg, T.E., Pitkala, K.H., 2006. Social contacts and their relationships to loneliness among aged people: A population-based study. Gerontology 52 (3), 181-187.

Russell, D.W., Cutrona, C.E., McRae, C., Gomez, M., 2012. Is Ioneliness the same as being alone? Journal of Psychology 146 (1-2), 7-22.

Scharf, T., Phillipson, C., Smith, A.E., 2005. Social exclusion of older people in deprived urban communities of England. European Journal of Ageing 2, 76-87.

Van Tilburg, T.G., Havens, B., De Jong Gierveld, J., 2004. Loneliness among older adults in the Netherlands, Italy and Canada: A multifaceted comparison. Canadian Journal on Aging 23, 169-180.

Weiss, R.S., 1973. Loneliness: The Experience of Emotional and Social Isolation. Cambridge, MA: The MIT Press. 